\title{
The relationships among sleep efficiency, pulmonary functions, and quality of life in patients with asthma
}

This article was published in the following Dove Press journal:

International Journal of General Medicine

I 3 November 2014

Number of times this article has been viewed

\author{
Akira Yamasaki' \\ Yuji Kawasaki \\ Kenichi Takeda' \\ Tomoya Harada' \\ Takehito Fukushima' \\ Miki Takata' \\ Kiyoshi Hashimoto' \\ Masanari Watanabe' \\ Jun Kurai ${ }^{1}$ \\ Koichi Nishimura ${ }^{3}$ \\ Eiji Shimizu'
}

'Division of Medical Oncology and Molecular Respirology, Department of Multidisciplinary Internal Medicine, Tottori University, Yonago, Japan;

${ }^{2}$ Division of Pulmonary Medicine, Tsuyama Daiichi Hospital, Tsuyama, Japan; ${ }^{3}$ Department of Pulmonary Medicine, National Center for Geriatrics and Gerontology, Obu, Japan
Correspondence: Akira Yamasaki Division of Medical Oncology and Molecular Respirology, Department of Multidisciplinary Internal Medicine, Tottori University, 36-I Nishi-cho, Yonago, Tottori, Japan

Email yamasaki@grape.med.tottori-u.ac.jp
Background: Sleep disturbance is commonly observed in patients with asthma, especially in those with poorly controlled asthma. Evaluating sleep quality to achieve good control of asthma is important since nocturnal asthmatic symptoms such as cough, wheezing, and chest tightness may disturb sleep. Actigraphy is an objective, ambulatory monitoring method for tracking a patient's sleep and wake activities and for assessing sleep quality, as reflected by total sleep time, sleep efficiency, duration of awakening after sleep onset (WASO), and sleep onset latency.

Patients and methods: Fifty patients with asthma were enrolled in this study. Sleep quality was assessed employing wristwatch-type actigraphy (Actiwatch 2). The level of asthma control was assessed by the Asthma Control Questionnaire (ACQ), and asthma-related quality of life was assessed by the Asthma Quality of Life Questionnaire (AQLQ). The parameters for sleep quality were compared using ACQ scores, AQLQ scores, and pulmonary function test results. Results: The total sleep time was 387.2 minutes, WASO was 55.8 minutes, sleep efficiency was $87.01 \%$, sleep onset latency was 8.17 minutes, and the average ACQ was 0.36 . Neither sleep efficiency nor WASO correlated with respiratory functions, ACQ scores, or AQLQ scores.

Conclusion: Sleep-related parameters assessed by actigraphy in well-controlled asthma do not correlate with pulmonary functions, the asthma control level, or daytime quality of life. Sleep quality should be evaluated independently when asthma is well-controlled.

Keywords: asthma control, respiratory function, sleep efficiency, actigraphy

\section{Introduction}

Sleep disorders are classified into seven major categories and 60 disorders are listed in the International Classification of Sleep Disorders. ${ }^{1}$ Seven major categories are: 1) insomnia; 2) sleep-related breathing disorders; 3) central disorders of hypersomnolence; 4) circadian rhythm sleep-wake disorders; 5) parasomnias; 6) sleep-related movement disorders; and 7) other sleep disorders. Sleep-related asthma is one of the disorders categorized into other sleep disorders, and the essential features of sleep-related asthma are the same as those of nocturnal asthma, such as awakening with dyspnea, wheezing, coughing, air hunger, or chest tightness during sleep. ${ }^{1}$

The prevalence of sleep disturbance is reportedly $12.5 \%-19.7 \%$ in adult asthmatic patients $^{2}$ and $38.95 \%$ in asthmatic children. ${ }^{3}$ Sleep disorders are known to be related to the severity of asthma and it has been reported that episodes of sleep-related asthma decrease in frequency and severity with effective treatment. ${ }^{4,5}$ However, recent studies have shown that sleep disorders are common in clinically stable asthmatic children. . $^{3,6}$ Hanson and Chen reported that asthma symptoms did not predict self-reported sleep quality. ${ }^{7}$ Furthermore, Catterall et al described sleep itself as an important factor in 
determining overnight bronchoconstriction. ${ }^{8}$ Therefore, evaluating sleep disorders in asthma patients is important for improving both asthmatic symptoms and quality of life.

In clinical practice, questionnaires such as the Asthma Quality of Life Questionnaire (AQLQ) and the Asthma Control Questionnaire (ACQ) are commonly used to assess quality of life and asthma control in patients with asthma. ${ }^{9}$ The health-related quality of life assessed by pediatric quality of life inventory is independent of asthma severity, ${ }^{10}$ and AQLQ scores did not correlate significantly with predicted forced expiratory volume in 1 second $\left(\mathrm{FEV}_{1}\right)$, forced vital capacity (FVC), or predicted FVC. ${ }^{11}$ Luyster et al reported that sleep quality is associated with poor asthma control and quality of life. $^{12}$

Recently, sleep evaluations by actigraphy in patients with a wide range of diseases, such as Alzheimer disease, ${ }^{13}$ Parkinson's disease, ${ }^{14,15}$ cancer, ${ }^{16}$ or rheumatoid arthritis, ${ }^{17}$ have been reported. Actigraphy is also used for evaluating sleep disturbance in allergic rhinitis. ${ }^{18,19}$ Krouse et al reported the usefulness of actigraphy for evaluating the relationship between degrees of asthma control, as assessed by ACQ and AQLQ, and sleep quality. ${ }^{20}$ They reported that uncontrolled asthma patients took longer to fall asleep, awoke more often, and spent more time awake during the night than those with controlled asthma. However, respiratory functions were not evaluated in their study.

In the present study, we examined pulmonary functions, quality of life, and quality of sleep employing actigraphy in patients with adult asthma to assess relationships between asthma control, as evaluated by pulmonary function tests and the questionnaire, and sleep quality.

\section{Patients and methods}

\section{Patients}

Patients diagnosed as having asthma by respiratory physicians according to the American Thoracic Society criteria ${ }^{21}$ participated in this study. Written informed consent was obtained from all patients. Those who had been diagnosed with obstructive sleep apnea syndrome were excluded from this study.

\section{Questionnaires}

To assess the level of disease control in the patients with asthma, they were asked to answer the ACQ. We also assessed the quality of life of these patients employing the AQLQ. Daytime sleepiness was assessed by the Epworth Sleepiness Scale (ESS). These three questionnaire series were applied to complete the assessments between the first and the second visit in this study.

\section{Assessing sleep quality by actigraphy}

The actigraph is a light-weight wrist accelerometer (Actiwatch 2; Philips Respironics, Murrysville, PA, USA). Actiwatch 2 has a light sensor and records a digitally-integrated measure of gross motor activity, which can be used to visualize rest and activity patterns, quality of physical activity, and sleep. The patients were asked to wear an actigraph for 7 consecutive days and their sleep qualities were assessed by Actiware 5 software. Activity counts (AC) were collected from Actiwatch 2. We defined 60 seconds as 1 epoch. Total activity counts were calculated by the following formula:

$$
\mathrm{A}=0.04 \mathrm{E}-2+0.020 \mathrm{E}-1+\mathrm{E}+0.2 \mathrm{E}+1+0.04 \mathrm{E}+2(1)
$$

where $\mathrm{A}=$ total $\mathrm{AC} ; \mathrm{E}=\mathrm{AC}$ during scored epoch; and $\mathrm{En}=\mathrm{AC}$ during previous or successive epochs. Sleep was defined as the period when total AC were less than or equal to 40 , which is regarded as the normal activity threshold for sleep analysis. ${ }^{22}$ We can assess total sleep time (minutes), sleep efficiency (\%), duration of awakenings after sleep onset (WASO) (minutes), and sleep onset latency (minutes) according to the Actiware 5 software.

\section{Measurement of respiratory functions and peak flow monitoring}

From January 2011 to August 2011, all patients were asked to perform pulmonary function tests with a Chestac 33 spirometer (Chest Co, Tokyo, Japan). The highest FVC and $\mathrm{FEV}_{1}$ values from a minimum of three pulmonary function tests were recorded. $\mathrm{FEV}_{1} \%$ is the $\mathrm{FEV}_{1}$ expressed as a percentage of the $\mathrm{FVC}\left(\mathrm{FEV}_{1} / \mathrm{FVC} \times 100\right) . \% \mathrm{FEV}_{1}$ is the $\mathrm{FEV}_{1}$ expressed as a percentage of predicted values for sex and height using reference equations from Berglund et al. ${ }^{23}$ All patients were also asked to measure the peak expiratory flow rate (PEFR) using a Mini-WRIGHT Peak-flow Meter (Clement Clarke, Harlow, UK). The patients recorded their PEFR twice a day, in the morning and in the evening, for 1 week and they wore an Actiwatch 2 to assess sleep quality during the same week while monitoring their PEFR.

\section{Statistical analysis}

GraphPad Prism 5 (GraphPad Software Inc., San Diego, CA, USA) was used for all statistical analyses. Values are 
presented as means \pm standard error of the mean. Spearman's correlation coefficients were used to examine correlations.

\section{Results}

\section{Characteristics of the patients}

In total, 50 patients were enrolled in this study. However, the results obtained from eleven subjects could not be analyzed due to missing actigraphic or PEFR data, such that the data from 39 patients were used. The average age, sex, height, weight, average $\%$ PEFR, and pulmonary function test values $\left(\% \mathrm{FVC}, \mathrm{FEV}_{1}, \% \mathrm{FEV}_{1}\right)$ are shown in Table 1.

\section{Actigraphy}

Total sleep time (minutes), WASO (minutes), sleep efficiency (\%), and sleep onset latency (minutes) were analyzed. The sleep time average was 387.2 minutes; WASO was 55.8 minutes; sleep efficiency was $83.0 \%$; and sleep onset latency was 8.1 minutes (Table 2).

\section{Relationships between sleep efficiency and pulmonary function tests}

We evaluated the relationships between sleep efficiency and pulmonary function tests. Trends were seen between sleep efficiency and $\% \mathrm{FVC}$ (Figure 1A), $\% \mathrm{FEV}_{1}$ (Figure 1B), $\mathrm{FEV}_{1} \%$ (Figure 1C), and \%PEFR (Figure 1D). Although there were slight tendencies for good $\mathrm{FEV}_{1} \%$ or $\%$ PEFR to be associated with good sleep efficiency, the relationships did not reach statistical significance.

\section{Relationships between WASO and pulmonary function tests}

Next, we evaluated the relationships between WASO and pulmonary function tests. WASO showed no relationships with \%FVC (Figure 2A), \% $\mathrm{FEV}_{1}$ (Figure 2B), $\mathrm{FEV}_{1} \%$ (Figure 2C), or \%PEFR (Figure 2D).

Table I Patient characteristics

\begin{tabular}{ll}
\hline Number of patients & 39 \\
Age $($ years $)$ & $57.4 \pm 16.1$ \\
Female/male & $25 / 14$ \\
Height $(\mathrm{cm})$ & $158.8 \pm 6.7$ \\
Weight $(\mathrm{kg})$ & $57.4 \pm 9.5$ \\
$\mathrm{BMI}\left(\mathrm{kg} / \mathrm{m}^{2}\right)$ & $22.7 \pm 3.1$ \\
Average PEFR $(\%)$ & $86.7 \pm 19.4$ \\
$\% \mathrm{FVC}\left(\mathrm{FVC} / \mathrm{FVC}{ }_{\text {pred }}\right)$ & $107.0 \pm 17.1$ \\
$\mathrm{FEV}_{1} \%\left(\mathrm{FEV}_{1} / \mathrm{FVC}\right)$ & $73.3 \pm 10.9$ \\
\%FEV $_{1}\left(\mathrm{FEV}_{1} / \mathrm{FEV}_{\text {। pred }}\right)$ & $98.8 \pm 20.4$ \\
\hline
\end{tabular}

Abbreviations: FVC, forced vital capacity; FEV, forced expiratory volume in I second; PEFR, peak expiratory flow rate; pred, predicted.
Table 2 Actigraphy data

\begin{tabular}{ll}
\hline Number of patients & 39 \\
Sleep time $(\mathrm{min})$ & $387.2 \pm 98.2$ \\
WASO $(\mathrm{min})$ & $55.81 \pm 22.68$ \\
Sleep efficiency $(\%)$ & $87.01 \pm 5.42$ \\
Sleep onset latency $(\mathrm{min})$ & $8.17 \pm 7.26$ \\
\hline
\end{tabular}

Abbreviation: WASO, duration of awakenings after sleep onset.

\section{Relationships of sleep efficiency with WASO and minimum morning PEFR/ recent best PEFR}

We calculated two PEFR indices from the consecutive 1-week PEFR monitoring results. One was the minimum morning value/maximum value which correlates with airway hyperresponsiveness. ${ }^{24}$ The other was the morning dip (maximum value minus minimum value)/evening value, which is associated with nocturnal asthma symptoms. ${ }^{25}$ We compared these indices with sleep efficiency and WASO but found no relationships (Figure 3).

\section{Epworth Sleepiness Scale and its relationship with sleep efficiency or WASO}

The average ESS was 4.95 and three patients scored above 11 , which is thought to raise the possibility of sleep apnea syndrome being present. The relationships of ESS with sleep efficiency (Figure 4A) and WASO (Figure 4B) were not significant.

\section{Correlations among ACQ score, sleep efficiency,WASO, and respiratory functions}

We assessed the relationships among the ACQ score, sleep efficiency, WASO, and respiratory functions. The ACQ score did not correlate with sleep efficiency or WASO. However, the ACQ score showed negative correlations with average PEFR, \%FVC, \% $\mathrm{FEV}_{1}$, and $\mathrm{FEV}_{1} \%$ (Table 3).

\section{Discussion}

We assessed sleep quality, pulmonary functions, and quality of life in patients with asthma. We found that sleep quality is not related to respiratory functions or quality of life in asthma patients. We also found the level of asthma control, as assessed by ACQ, to not be associated with sleep quality, though patients with severe airflow limitation tended to show lower sleep efficiency and longer WASO than those with mild and moderate airflow limitations. 

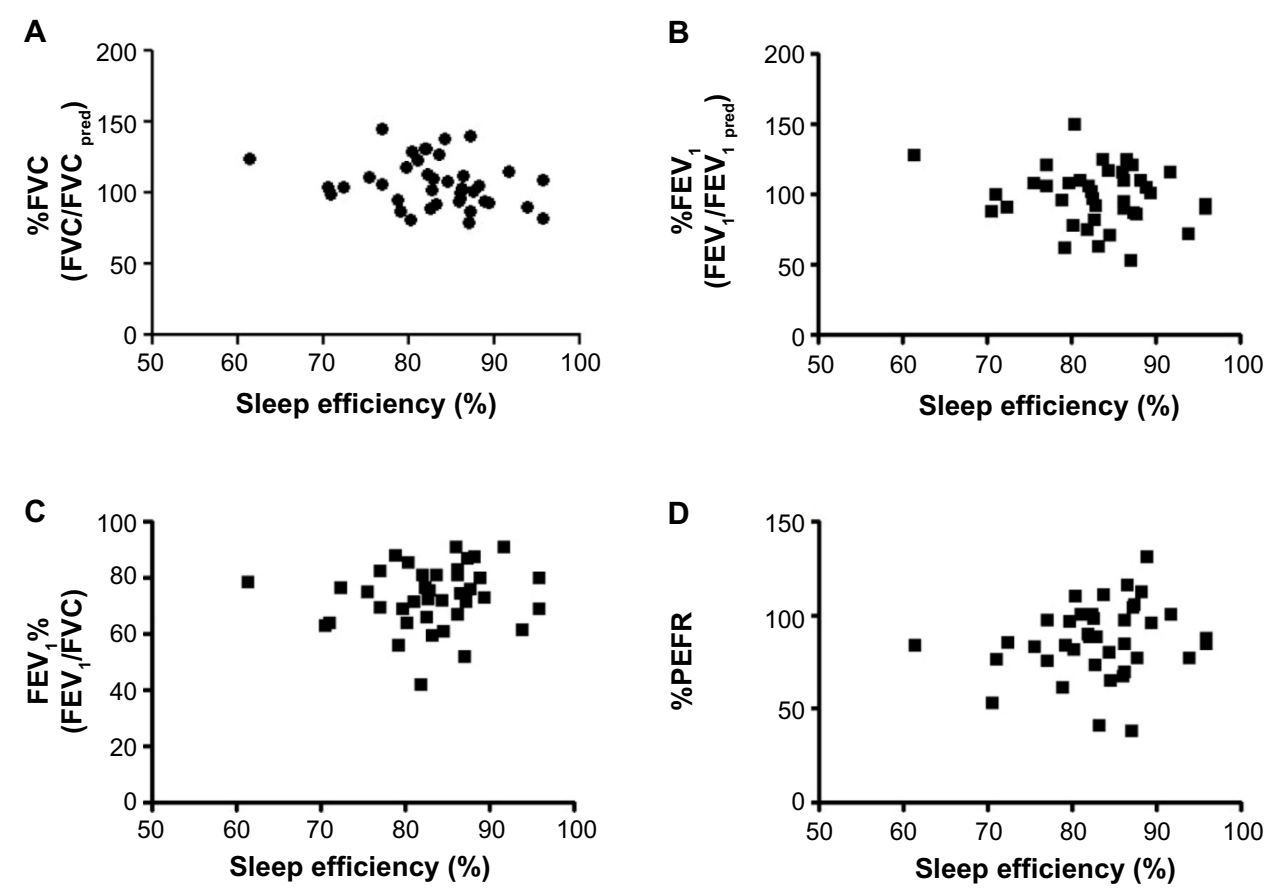

Figure I Correlations between sleep efficiency and pulmonary function tests.

Notes: Sleep efficiency shows no correlation with (A) \%FVC, (B) \%FEV, (C) FEV \%, or (D) \%PEFR

Abbreviations: FEV $_{1}$, forced expiratory volume in I second; PEFR, peak expiratory flow rate; pred, predicted; FVC, forced vital capacity.

Krouse et al, using wrist actigraphy in ten patients with asthma, reported that the degree of asthma control correlated strongly with sleep quality. They showed that ACQ scores correlated positively with sleep onset latency, which means that poorly controlled asthma patients take a long time to fall asleep. ${ }^{20}$ They also showed correlations between ACQ scores or AQLQ scores and sleep efficiency, number of sleep episodes, and total time awake. In our study, neither ACQ scores nor AQLQ scores correlated significantly with any of the several parameters related to sleep quality. These
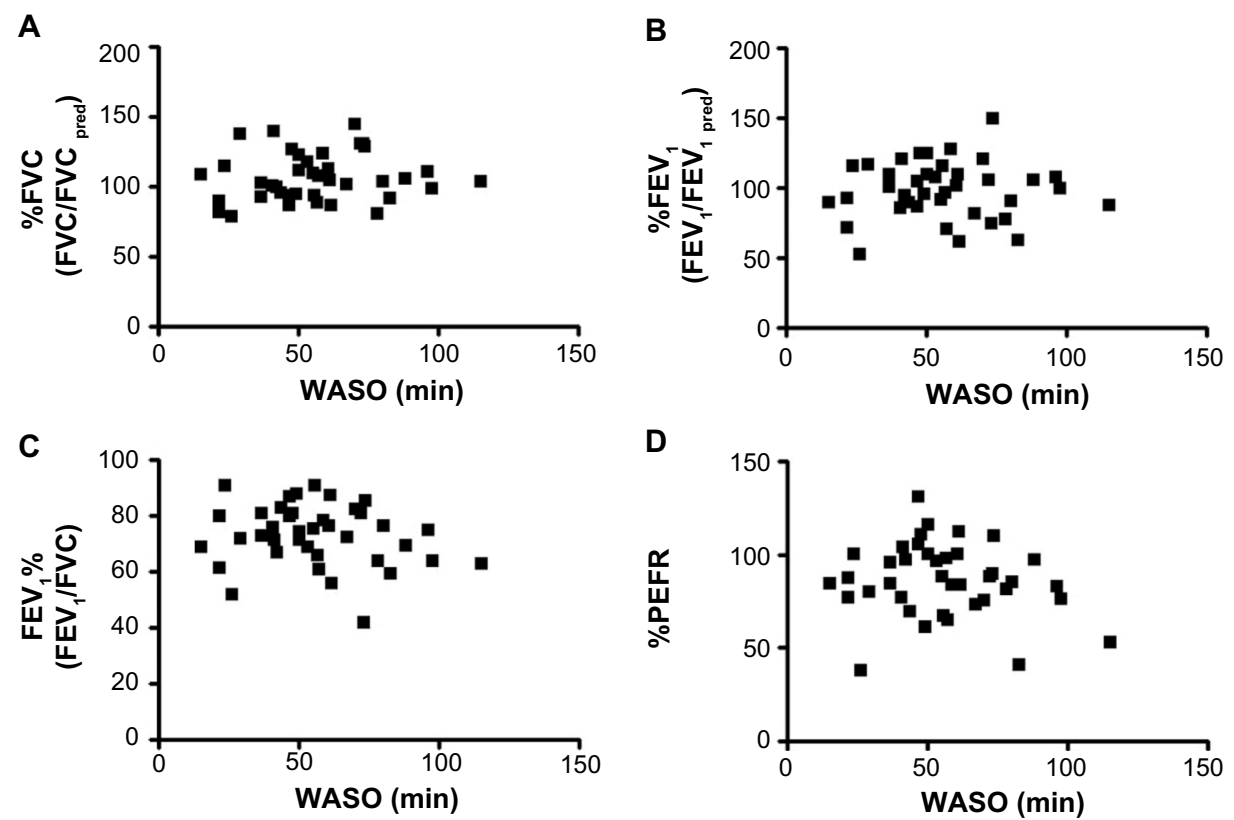

Figure 2 Correlations between WASO and pulmonary function tests.

Notes: WASO shows no correlation with \%FVC (A), \%FEV, (B), FEV \% (C), or (D) \%PEFR.

Abbreviations: $\mathrm{FEV}_{1}$, forced expiratory volume in I second; PEFR, peak expiratory flow rate; pred, predicted; FVC, forced vital capacity; WASO, duration of awakenings after sleep onset. 

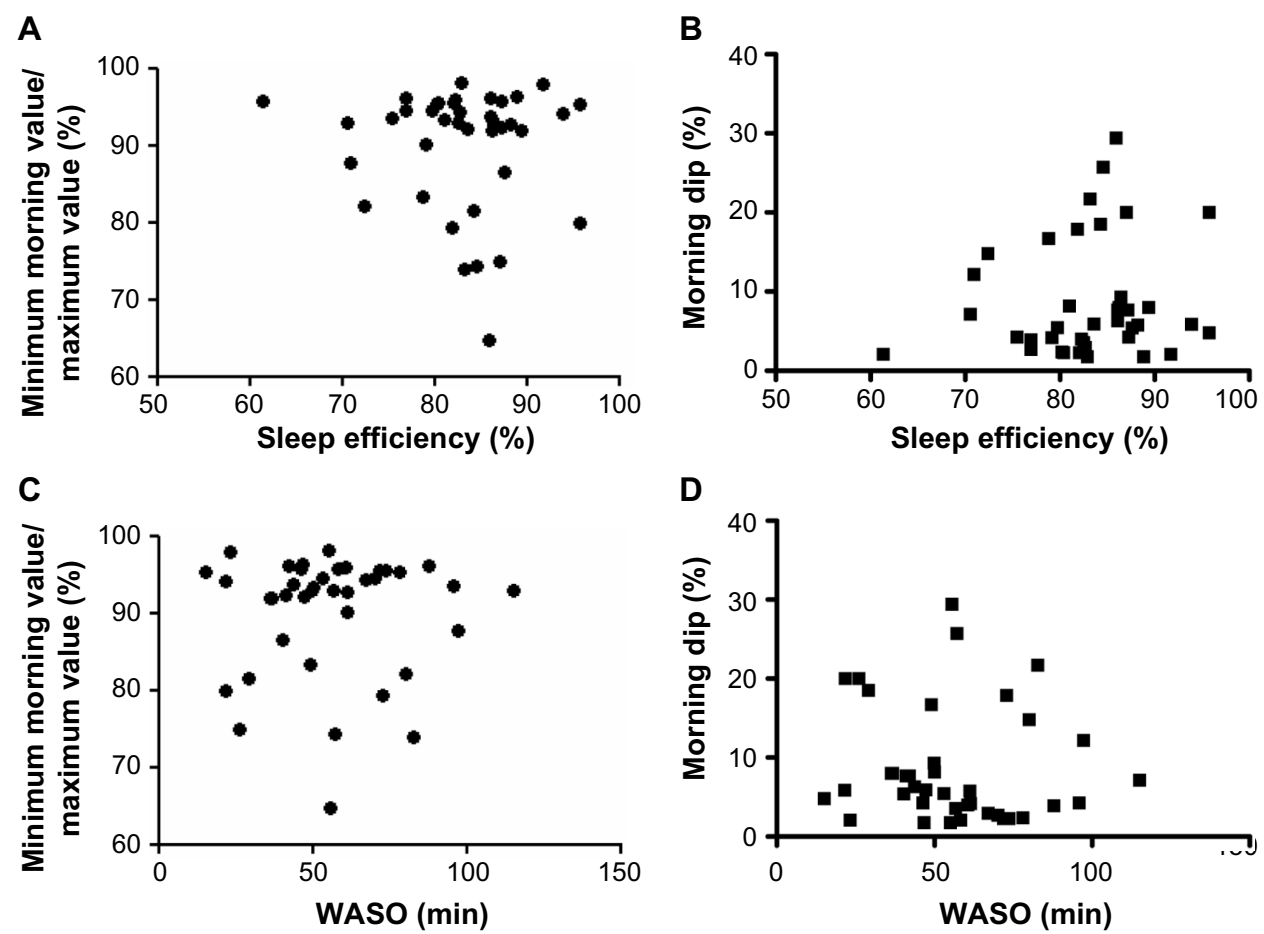

Figure 3 Correlations between sleep efficiency or WASO and minimum morning value/maximum value or morning dip in PEFR.

Notes: Sleep efficiency shows no correlation with minimum morning value/maximum value (A) and morning dip (B). WASO also shows no correlations with minimum morning value/maximum value (C) and morning dip (D).

Abbreviations: PEFR, peak expiratory flow rate; WASO, duration of awakenings after sleep onset.

parameters also showed a negative correlation between ACQ and AQLQ. We also found that ACQ and AQLQ showed a significant correlation, though that of ACQ was very low (data not shown). In our study, the level of asthma control was very good according to the average ACQ scores (0.36). On the other hand, the level of asthma control was worse (ACQ 2.20 at first visit and 1.93 at second visit) in Krouse et al's study. ${ }^{20}$ This discrepancy is thus attributable to the difference in asthma control levels between the two study groups.

Many patients with asthma reportedly have daytime sleepiness. ${ }^{26,27}$ Mastronarde et $\mathrm{al}^{27}$ studied sleep disturbance in symptomatic asthma patients using the ESS. They

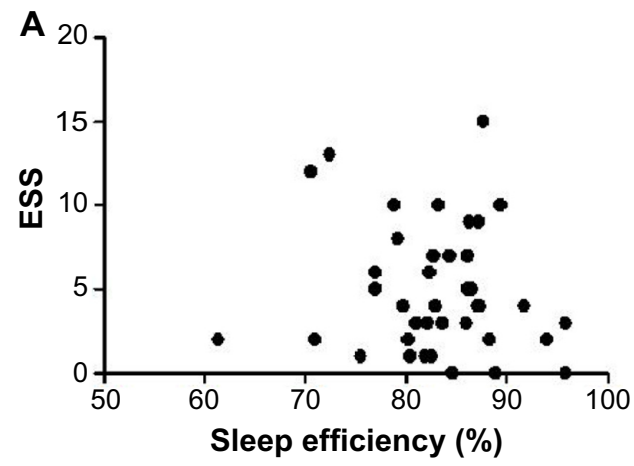

showed that ESS scores averaged $8.5 \pm 4.9$ in 487 subjects. Krouse et al also reported that the average ESS score was 9.20 and that four of their ten patients $(40 \%)$ had scores over $10 .{ }^{20}$ In our study, the average ESS score was low $(4.95 \pm 3.81)$ and only three of the 39 patients $(7.6 \%)$ had scores over 10. Furthermore, there was no significant correlation between ESS scores and either sleep efficiency or WASO. These results suggested that our study subjects were well controlled in terms of nocturnal symptoms and that subjects with obstructive sleep apnea syndrome were apparently excluded, despite the prevalence of obstructive sleep apnea in asthma being high, ${ }^{28}$ especially in patients with severe asthma..$^{29,30}$

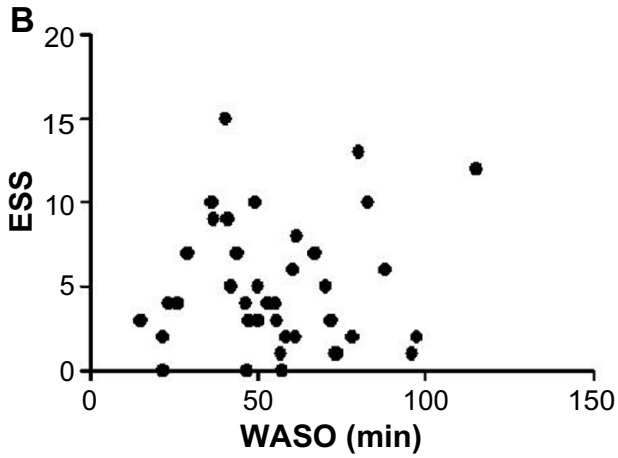

Figure 4 Correlation between ESS and sleep efficiency or WASO.

Note: ESS correlates with neither sleep efficiency (A) nor WASO (B).

Abbreviations: ESS, Epworth Sleepiness Scale; WASO, duration of awakenings after sleep onset. 
Table 3 Correlation of ACQ and AQLQ with sleep efficiency, WASO, and respiratory functions

\begin{tabular}{|c|c|c|}
\hline & $A C Q$ & AQLQ \\
\hline Sleep efficiency (\%) & 0.1169 & -0.0359 \\
\hline WASO (min) & 0.1817 & 0.1280 \\
\hline Mean \%PEFR & $-0.3657 *$ & $0.4744 * *$ \\
\hline$\% F V C\left(F V C / F V C{ }_{\text {pred }}\right)$ & $-0.4933^{* *}$ & $0.5293 * *$ \\
\hline$\% \mathrm{FEV}_{1}\left(\mathrm{FEV}_{1} / \mathrm{FEV}_{1 \text { pred }}\right)$ & $-0.5560 * *$ & $0.4449 * *$ \\
\hline $\mathrm{FEV}_{1} \%(\mathrm{FEV}, / \mathrm{FVC})$ & $-0.3454^{*}$ & 0.1591 \\
\hline
\end{tabular}

Notes: $* P<0.05$. $* * P<0.01$.

Abbreviations: ACQ, Asthma Control Questionnaire; AQLQ, Asthma Quality of Life Questionnaire; $\mathrm{FEV}_{1}$, forced expiratory volume in I second; FVC, forced vital capacity; PEFR, peak expiratory flow rate; pred, predicted; WASO, duration of awakenings after sleep onset.

Luyster et al reported that poor sleep quality is an independent predictor of worse asthma control and quality of life in both nonsevere and severe asthma patients. ${ }^{12}$ In their study, sleep quality was assessed employing Pittsburgh Sleep Quality Index scores, which are derived from a selfreported questionnaire designed to measure sleep quality for the past 1 month. ${ }^{31}$ They reported that poor sleep quality is associated with poor asthma control but not nighttime asthma disturbance. Braido et al also suggested that in real life, poor sleep might be independent of nighttime asthma symptoms even when asthma is well controlled. ${ }^{32}$ Therefore, our data related to sleep may represent the actual sleep situation of patients with asthma which is well controlled.

In this study, we assessed the correlations among pulmonaryfunction parameters ( $\% \mathrm{FVC}, \% \mathrm{FEV}_{1}, \mathrm{FEV}_{1} \%$, and $\left.\mathrm{PEFR}\right)$, sleep quality parameters (sleep efficiency and WASO), and AQLQ. We found no relationships between the parameters of sleep and asthma-related quality of life. Furthermore, pulmonary functions correlated with neither sleep efficiency nor WASO. However, asthma-related quality of life and pulmonary-function parameters showed significant positive correlations. These results suggest pulmonary functions to possibly be related to daytime quality of life, but not sleep quality in patients with asthma. Since health-related quality of life reportedly shows only a weak correlation with pulmonary-function parameters in patients with asthma, ${ }^{33,34}$ sleep quality should be assessed individually based on pulmonary function tests and the AQLQ.

Chronic obstructive pulmonary disease (COPD) is a common preventable and treatable disease, characterized by persistent airflow limitation. ${ }^{35}$ Sleep disturbance in COPD is common $^{36,37}$ and associated with oxygen desaturation, cardiac arrhythmia, pulmonary hypertension, and nocturnal death. ${ }^{38}$ Omachi et al reported that sleep disturbance was associated with cough, dyspnea, and COPD severity. They have shown that sleep disturbance predicted COPD exacerbations, emergency room visits, and poorer survival. ${ }^{39}$ These findings are different from previous studies for patients with asthma. However, patients with asthma who are elderly and have smoking history sometimes show features of COPD (asthma-COPD overlap syndrome).$^{40}$ Since patients with asthma-COPD overlap syndrome might show more sleep disturbance compared to patients with asthma or COPD alone, further studies are needed to assess the relationship among sleep quality, pulmonary functions, exacerbations, and mortality in these patients. ${ }^{40}$

The total sleep time in patients with asthma in this study averaged 387.2 minutes. The average sleep time of nonasthmatic patients was 389.9 minutes, ${ }^{41}$ as assessed by actigraphy. The average sleep time of Japanese subjects studied is 413 minutes, as assessed by questionnaire, ${ }^{42}$ while patients with nocturnal asthma had shorter sleep times than normal subjects. ${ }^{43}$ Although different methods for evaluating sleep might influence actual total sleep time, the total sleep time of our study subjects is likely to be similar to that of nonasthmatic patients. Kripke et al investigated the relationship between mortality and actigraphic sleep time. ${ }^{44}$ They adjusted for survival functions and estimated that $61 \%$ of subjects slept less than 300 minutes and that survival was $78 \%$ for subjects sleeping longer than 390 minutes. The subjects with sleep durations ranging from 300 to 390 minutes had a survival rate of $90 \%$. The actigraphic sleep time of the patients in this study appears to be favorable, but further studies are needed to determine whether sleep time influences mortality in subjects with well-controlled asthma.

A limitation of this study is that we failed to evaluate comorbidities in our asthma patients. In adult patients with asthma, nasal congestion due to rhinitis, obesity, and smoking all impact the risk of insomnia. ${ }^{45}$ Furthermore, asthmaassociated comorbidities, such as sleep apnea syndrome and gastroesophageal reflux, influence both asthma severity and sleep disorders. ${ }^{5,12,46,47}$ We were also unable to assess drug side-effects, including those of oral corticosteroids, methylxanthine, antidepressants, and hypnotic drugs, which can affect sleep quality. We thought that the duration of the study period might be too short to evaluate sleep quality in well-controlled asthma. Further study is necessary to clarify these points.

\section{Conclusion}

In conclusion, sleep quality in well-controlled asthma is not related to parameters of pulmonary function or to assessments of asthma-associated quality of life. Sleep disturbance might be related not only to asthma control but also to other conditions such as comorbidities or drugs. ${ }^{32}$ Among patients 
with asthma, sleep quality should be assessed independently when asthma is well controlled.

\section{Disclosure}

The authors report no conflicts of interest in this work.

\section{References}

1. American Academy of Sleep Medicine International Classification of Sleep Disorders, 3rd edition. American Academy of Sleep Medicine, Darien, IL, USA. 2014.

2. Liu Y, Croft JB, Wheaton AG, et al. Association between perceived insufficient sleep, frequent mental distress, obesity and chronic diseases among US adults, 2009 behavioral risk factor surveillance system. BMC Public Health. 2013;13:84.

3. Chugh IM, Khanna P, Shah A. Nocturnal symptoms and sleep disturbances in clinically stable asthmatic children. Asian Pac J Allergy Immunol. 2006;24:135-142.

4. Montplaisir J, Walsh J, Malo JL. Nocturnal asthma: features of attacks, sleep and breathing patterns. Am Rev Respir Dis. 1982;125:18-22.

5. Ballard RD. Sleep and medical disorders. Prim Care. 2005;32:511-533.

6. Daniel LC, Boergers J, Kopel SJ, Koinis-Mitchell D. Missed sleep and asthma morbidity in urban children. Ann Allergy Asthma Immunol. 2012;109:41-46

7. Hanson MD, Chen E. The temporal relationships between sleep, cortisol, and lung functioning in youth with asthma. J Pediatr Psychol. 2008;33:312-316.

8. Catterall JR, Rhind GB, Stewart IC, Whyte KF, Shapiro CM, Douglas NJ. Effect of sleep deprivation on overnight bronchoconstriction in nocturnal asthma. Thorax. 1986;41:676-680.

9. Juniper EF, Guyatt GH, Epstein RS, Ferrie PJ, Jaeschke R, Hiller TK. Evaluation of impairment of health related quality of life in asthma: development of a questionnaire for use in clinical trials. Thorax. 1992;47:76-83.

10. Hallstrand TS, Curtis JR, Aitken ML, Sullivan SD. Quality of life in adolescents with mild asthma. Pediatr Pulmonol. 2003;36:536-543.

11. Moy ML, Israel E, Weiss ST, et al. Clinical predictors of health-related quality of life depend on asthma severity. Am J Respir Crit Care Med. 2001;163:924-929.

12. Luyster FS, Teodorescu M, Bleecker E, et al. Sleep quality and asthma control and quality of life in non-severe and severe asthma. Sleep Breath. 2012;16:1129-1137.

13. Kawada T. Sleep evaluation by actigraphy for patients with Alzheimer disease. JAMA Neurol. 2013;70:1074.

14. Maglione JE, Liu L, Neikrug AB, et al. Actigraphy for the assessment of sleep measures in Parkinson's disease. Sleep. 2013;36: 1209-1217.

15. Pan W, Kwak S, Li F, et al. Actigraphy monitoring of symptoms in patients with Parkinson's disease. Physiol Behav. 2013;119:156-160.

16. Mustian KM, Sprod LK, Janelsins M, et al. Multicenter, randomized controlled trial of yoga for sleep quality among cancer survivors. $J$ Clin Oncol. 2013;31(26):3233-3241.

17. Clarke LL, Wilson S, Kirwan JR. Using actigraphy to measure sleep patterns in rheumatoid arthritis: a pilot study in patients taking nighttime prednisone. Musculoskeletal Care. 2013;11(3):179-185.

18. Rimmer J, Downie S, Bartlett DJ, Gralton J, Salome C. Sleep disturbance in persistent allergic rhinitis measured using actigraphy. Ann Allergy Asthma Immunol. 2009;103:190-194.

19. Yuksel H, Sogut A, Yilmaz H, Yilmaz O, Dinc G. Sleep actigraphy evidence of improved sleep after treatment of allergic rhinitis. Ann Allergy Asthma Immunol. 2009;103:290-294.

20. Krouse HJ, Yarandi H, McIntosh J, Cowen C, Selim V. Assessing sleep quality and daytime wakefulness in asthma using wrist actigraphy. J Asthma. 2008;45:389-395.
21. No authors listed. Standards for the diagnosis and care of patients with chronic obstructive pulmonary disease (COPD) and asthma. This official statement of the American Thoracic Society was adopted by the ATS Board of Directors, November 1986. Am Rev Respir Dis. 1987;136:225-244.

22. Hyde M, O’Driscoll DM, Binette S, et al. Validation of actigraphy for determining sleep and wake in children with sleep disordered breathing. J Sleep Res. 2007;16:213-216.

23. Berglund E, Birath G, Bjure J, et al. Spirometric studies in normal subjects. I. Forced expirograms in subjects between 7 and 70 years of age. Acta Med Scand. 1963;173:185-192.

24. Reddel HK, Salome CM, Peat JK, Woolcock AJ. Which index of peak expiratory flow is most useful in the management of stable asthma? Am J Respir Crit Care Med. 1995;151:1320-1325.

25. Bellia V, Cuttitta G, Cibella F, et al. Effect of ageing on peak expiratory flow variability and nocturnal exacerbations in bronchial asthma. Eur Respir J. 1997;10:1803-1808.

26. Vir R, Bhagat R, Shah A. Sleep disturbances in clinically stable young asthmatic adults. Ann Allergy Asthma Immunol. 1997;79:251-255.

27. Mastronarde JG, Wise RA, Shade DM, Olopade CO, Scharf SM; American Lung Association Asthma Clinical Research Centers. Sleep quality in asthma: results of a large prospective clinical trial. JAsthma. 2008;45:183-189.

28. Braido F, Baiardini I, Lacedonia D, et al. Sleep Apnea Risk in Patients With Asthma With or Without Comorbid Rhinitis. Respir Care. Epub January 10, 2014.

29. Julien JY, Martin JG, Ernst P, et al. Prevalence of obstructive sleep apnea-hypopnea in severe versus moderate asthma. J Allergy Clin Immunol. 2009; 124:371-376.

30. Guven SF, Dursun AB, Ciftci B, Erkekol FO, Kurt OK. The prevalence of obstructive sleep apnea in patients with difficult-to-treat asthma. Asian Pac J Allergy Immunol. 2014;32:153-159.

31. Buysse DJ, Reynolds CF 3rd, Monk TH, Berman SR, Kupfer DJ. The Pittsburgh Sleep Quality Index: a new instrument for psychiatric practice and research. Psychiatry Res. 1989;28:193-213.

32. Braido F, Baiardini I, Ghiglione V, et al. Sleep disturbances and asthma control: a real life study. Asian Pac J Allergy Immunol. 2009;27:27-33.

33. Juniper EF. Health-related quality of life in asthma. Curr Opin Pulm Med. 1999;5:105-110.

34. Takeda T, Oga T, Niimi A, et al. Relationship between small airway function and health status, dyspnea and disease control in asthma. Respiration. 2010;80:120-126.

35. Global Initiative for Chronic Obstructive Pulmonary Disease (GOLD). Global Strategy for Diagnosis, Management and Prevention of COPD. 2014. Available from: http://www.goldcopd.org/uploads/users/files/ GOLD_Report_2014_Jan23.pdf. Accessed August 27, 2014.

36. Agusti A, Hedner J, Marin JM, Barbé F, Cazzola M, Rennard S. Night-time symptoms: a forgotten dimension of COPD. Eur Respir Rev. 2011;20:183-194.

37. McSharry DG, Ryan S, Calverley P, Edwards JC, McNicholas WT. Sleep quality in chronic obstructive pulmonary disease. Respirology. 2012;17:1119-1124.

38. McNicholas WT, Verbraecken J, Marin JM. Sleep disorders in COPD: the forgotten dimension. Eur Respir Rev. 2013;22:365-375.

39. Omachi TA, Blanc PD, Claman DM, et al. Disturbed sleep among COPD patients is longitudinally associated with mortality and adverse COPD outcomes. Sleep Med. 2012;13:476-483.

40. Diagnosis of Diseases of Chronic Airflow Limitation: Asthma COPD and Asthma-COPD Overlap Syndrome (ACOS). Based on the Global Strategy for Asthma Management and Prevention and the Global Strategy for the Diagnosis, Management and Prevention of Chronic Obstructive Pulmonary Disease. 2014. Available from: http://www. ginasthma.org/documents/14. Accessed August 27, 2014.

41. Fung MM, Peters K, Ancoli-Israel S, Redline S, Stone KL, Barrett-Connor E. Total sleep time and other sleep characteristics measured by actigraphy do not predict incident hypertension in a cohort of community-dwelling older men. J Clin Sleep Med. 2013;9: 585-591. 
42. Soldatos CR, Allaert FA, Ohta T, Dikeos DG. How do individuals sleep around the world? Results from a single-day survey in ten countries. Sleep Med. 2005;6:5-13.

43. Fitzpatrick MF, Engleman H, Whyte KF, Deary IJ, Shapiro CM, Douglas NJ. Morbidity in nocturnal asthma: sleep quality and daytime cognitive performance. Thorax. 1991;46:569-573.

44. Kripke DF, Langer RD, Elliott JA, Klauber MR, Rex KM. Mortality related to actigraphic long and short sleep. Sleep Med. 2011;12:28-33.
45. Sundbom F, Lindberg E, Bjerg A, et al. Asthma symptoms and nasal congestion as independent risk factors for insomnia in a general population: results from the GA(2)LEN survey. Allergy. 2013;68:213-219.

46. Prasad B, Nyenhuis SM, Weaver TE. Obstructive sleep apnea and asthma: associations and treatment implications. Sleep Med Rev. 2014; 18(2):165-171.

47. Boulet LP, Boulay MÈ. Asthma-related comorbidities. Expert Rev Respir Med. 2011;5:377-393.

\section{Publish your work in this journal}

The International Journal of General Medicine is an international, peer-reviewed open-access journal that focuses on general and internal medicine, pathogenesis, epidemiology, diagnosis, monitoring and treatment protocols. The journal is characterized by the rapid reporting of reviews, original research and clinical studies across all disease areas.
A key focus is the elucidation of disease processes and management protocols resulting in improved outcomes for the patient. The manuscript management system is completely online and includes a very quick and fair peer-review system. Visit http://www.dovepress.com/ testimonials.php to read real quotes from published authors.

Submit your manuscript here: http://www.dovepress.com/international-journal-of-general-medicine-journal 